\title{
A COUNTEREXAMPLE TO THE BOUNDED ORBIT CONJECTURE ${ }^{1}$
}

\author{
BY
}

\author{
STEPHANIE M. BOYLES
}

\begin{abstract}
A long outstanding problem in the topology of Euclidean spaces is the Bounded Orbit Conjecture, which states that every homeomorphism of $E^{2}$ onto itself, with the property that the orbit of every point is bounded, must have a fixed point. It is well known that the conjecture is true for orientation preserving homeomorphisms. We provide a counterexample to the conjecture by constructing a fixed point free orientation reversing homeomorphism which satisfies the hypothesis of the conjecture.
\end{abstract}

1. Introduction. In 1912 L. E. J. Brouwer proved his famous translation theorem [7] which states that if $h$ is an orientation preserving homeomorphism of $E^{2}$ onto itself having no fixed points, then $h$ is a translation. By a translation, Brouwer meant that for each $x$ in $E^{2}, h^{n}(x) \rightarrow \infty$ as $n \rightarrow \pm \infty$; that is, the orbit of every point is unbounded. Thus, if $h$ is an orientation preserving homeomorphism of $E^{2}$ onto itself such that the orbit of some point is bounded, then $h$ must have a fixed point. Obviously, Brouwer's theorem cannot be true for orientation reversing homeomorphisms. For instance, let $h$ be a reflection across the $y$-axis followed by the upward shift $f(x, y)=(x, y-|x|+1)$ in the strip determined by $|x|<1$ (Figure 1). Then every point $(x, y)$ with $|x| \geqslant 1$ has bounded orbit, but $h$ is fixed point free!

The question arose as to whether or not a homeomorphism of $E^{2}$ onto itself with the property that the orbit of every point is bounded must have a fixed point. This eventually became known as the bounded orbit problem, and a considerable amount of research relating to this problem has accumulated in the literature [1]-[3], [5]-[12]. The objective of this paper is to construct a fixed point free orientation reversing homeomorphism of $E^{2}$ onto itself with the property that the orbit of every point is bounded. By modifying an example of Bing's [4], Brechner and Mauldin [5] gave an example of an orientation preserving homeomorphism of $E^{3}$ onto itself which is fixed point free and such that the orbit of every point is bounded. We note that this result follows as an easy corollary from the example we construct. The homeomorphism $h$ constructed in our example has the property that

Received by the editors February 12, 1980 and, in revised form, May 6, 1980; presented to the Society, January 7, 1981.

1980 Mathematics Subject Classification. Primary 54H25, 55M20.

Key words and phrases. Fixed point free homeomorphism, bounded orbits, orientation preserving, orientation reversing.

'This is part of the author's doctoral dissertation at the University of Florida under the supervision of Professor Gerhard X. Ritter. 
any arc connecting $x$ with $h(x)$ has unbounded orbit. In fact, it follows from a theorem of Bell's [3] that any counterexample to the bounded orbit conjecture must exhibit this property.

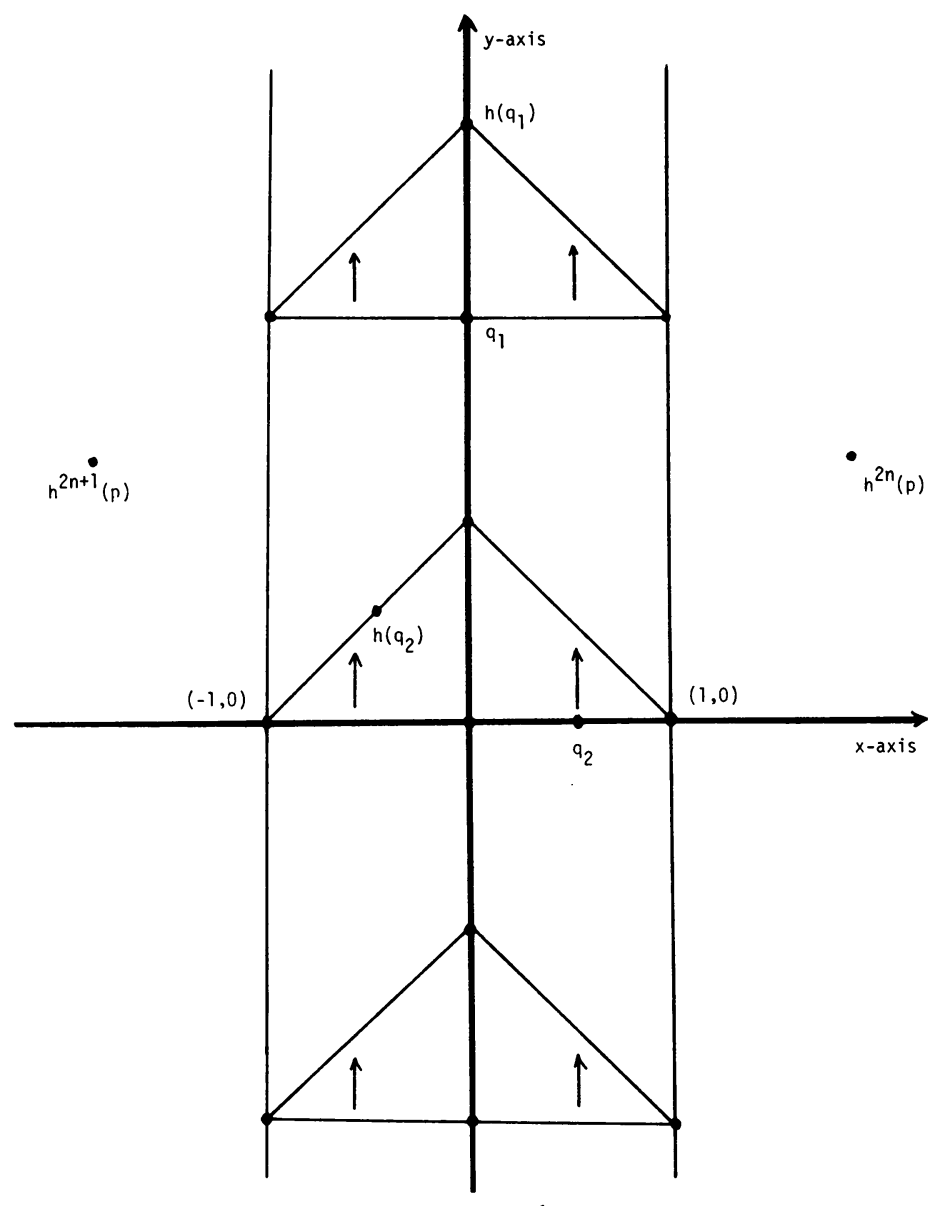

Figure 1

2. Notation and definitions. The letters $p, q, v$ are used to denote points in $E^{2}$. Line segments and open arcs are denoted by $S$ and $A$, respectively. The letter $t$ is always a real number between 0 and 1 ; and $i, j, k, m, n$ take only integer values. We let $x$ and $y$ correspond to the $x$ and $y$ coordinates of a point in $E^{2}$.

The orbit of a point $p, O(p)$, is defined as the set of all $h^{k}(p)$, where $k$ ranges over the integers.

Let $v_{1}, v_{2}, \ldots, v_{n}$ be $n$ points in general position in $E^{k}$. Denote the closed convex hull of $\left\{v_{1}, v_{2}, \ldots, v_{n}\right\}$ by $\left[v_{1}, v_{2}, \ldots, v_{n}\right]$. By a linear map we shall mean a map

$$
h:\left[v_{1}, v_{2}, \ldots, v_{n}\right] \rightarrow\left[h\left(v_{1}\right), h\left(v_{2}\right), \ldots, h\left(v_{n}\right)\right]
$$

defined by

$$
\sum_{i=1}^{n} t_{i} v_{i} \rightarrow \sum_{i=1}^{n} t_{i} h\left(v_{i}\right), \quad \text { where } \sum_{i=1}^{n} t_{i}<1
$$


3. Definition of $h$. Let $B$ denote the open strip $\{(x, y):|x|<1\}$. If $p$ is not in $B$, define $h(p)=h(x, y) \equiv(-x, y)$, where $\equiv$ indicates definition. Thus, on the complement of $B$ the map is a reflection across the $y$-axis.

To describe the homeomorphism on $B$, we first define $h$ on a countable set of points which will be the vertices of convex polygons in $B$. For all $m \geqslant 0$ and $k \geqslant 1$, let

$$
v_{ \pm m, 0}=\left(\frac{ \pm m}{m+1}, 0\right), \quad \text { and } \quad v_{ \pm m, k}=\left(\frac{ \pm m}{m+1}, \sum_{i=1}^{k} \frac{1}{m+i}\right) \text {. }
$$

For all $j$ and $k \geqslant 0$, define

$$
h\left(v_{j, k}\right) \equiv v_{(-1)^{k+1}-j, k+1} .
$$

Denote $\left[v_{j-1, k}, v_{j, k}\right]$ by $S_{j, k}$. Extend $h$ linearly on $S_{j, k}$ by defining

$$
\begin{aligned}
h\left(S_{j, k}\right) & =h\left(\left[v_{j-1, k}, v_{j, k}\right]\right) \\
& \equiv\left[h\left(v_{j-1, k}\right), h\left(v_{j, k}\right)\right], \quad \text { for all } j, k \geqslant 0 .
\end{aligned}
$$

Let $A=\{(x, y):|x|<1$ and $y=0\}$. Thus, $h^{k}(A)=\cup_{j=-\infty}^{\infty} S_{j, k}$ (Figure 2). Note that the positive orbit of $A$ forms an unbounded sequence of arcs contained in the half of $B$ having nonnegative $y$-coordinate, and that the intersection of any two of these arcs is empty. One may view the homeomorphism $h$ on $h^{k}(A), k \geqslant 0$, as the following composition: $h=\pi \cdot r \cdot s$, where $s$ is a shift of every segment $S_{j, k}$ either "left" one segment if $k$ is odd (i.e. for all $j, S_{j, k} \rightarrow S_{j-1, k}$ ), or "right" one segment if $k$ is even (i.e. for all $j, S_{j, k} \rightarrow S_{j+1, k}$ ), $r$ : a reflection through the $y$-axis, and $\pi$ is an upward projection to $h^{k+1}(A)$.

To describe $h$ on the regions between $h^{k-1}(A)$ and $h^{k}(A), k \geqslant 0$, consider the following two sequences of points on each vertical segment $\left[v_{j, k-1}, v_{j, k}\right]$ :

$$
\begin{aligned}
v_{j, k}^{1 / n} & \equiv v_{j, k-1}+\left(v_{j, k}-v_{j, k-1}\right) / n, \quad \text { where } n \geqslant 3, \quad \text { and } \\
v_{j, k}^{(n-1) / n} & \equiv v_{j, k-1}+\left(v_{j, k}-v_{j, k-1}\right)(n-1) / n, \quad \text { where } n \geqslant 2 .
\end{aligned}
$$

Note that $\left\{v_{j, k}^{1 / n}\right\}_{n \geqslant 3}$ and $\left\{v_{j, k}^{(n-1) / n}\right\}_{n \geqslant 2}$ are sequences converging to $v_{j, k-1}$ and $v_{j, k}$, respectively. Let

$$
S_{j, k}^{t}=\left[v_{j-1, k}^{t}, v_{j, k}^{t}\right], \text { and } A_{k, t}=\bigcup_{j=-\infty}^{\infty} S_{j, k}^{t},
$$

where $t=1 / n$ for $n \geqslant 3$, or $t=(n-1) / n$ for $n \geqslant 2$. Thus, for every positive integer $k,\left\{A_{k, 1 / n}\right\}_{n \geqslant 3}$ and $\left\{A_{k,(n-1) / n}\right\}_{n \geqslant 2}$ are disjoint sequences of open arcs between $h^{k-1}(A)$ and $h^{k}(A)$ limiting to $h^{k-1}(A)$ and $h^{k}(A)$, respectively. For $k \geqslant 1$, and for all $j$, define

$$
\begin{aligned}
h\left(v_{j, k}^{1 / n}\right) & \equiv v_{(-1)^{k}-j, k+1}^{1 /(n-2)}, \quad \text { for } n \geqslant 4, \\
h\left(v_{j, k}^{1 / 3}\right) & \equiv v_{-j, k+1}^{2 / 3}, \quad \text { and } \\
h\left(v_{j, k}^{(n-1) / n}\right) & \equiv v_{(-1)^{k}}^{(n+1) /(n+2)}, \quad \text { for } n \geqslant 2 .
\end{aligned}
$$




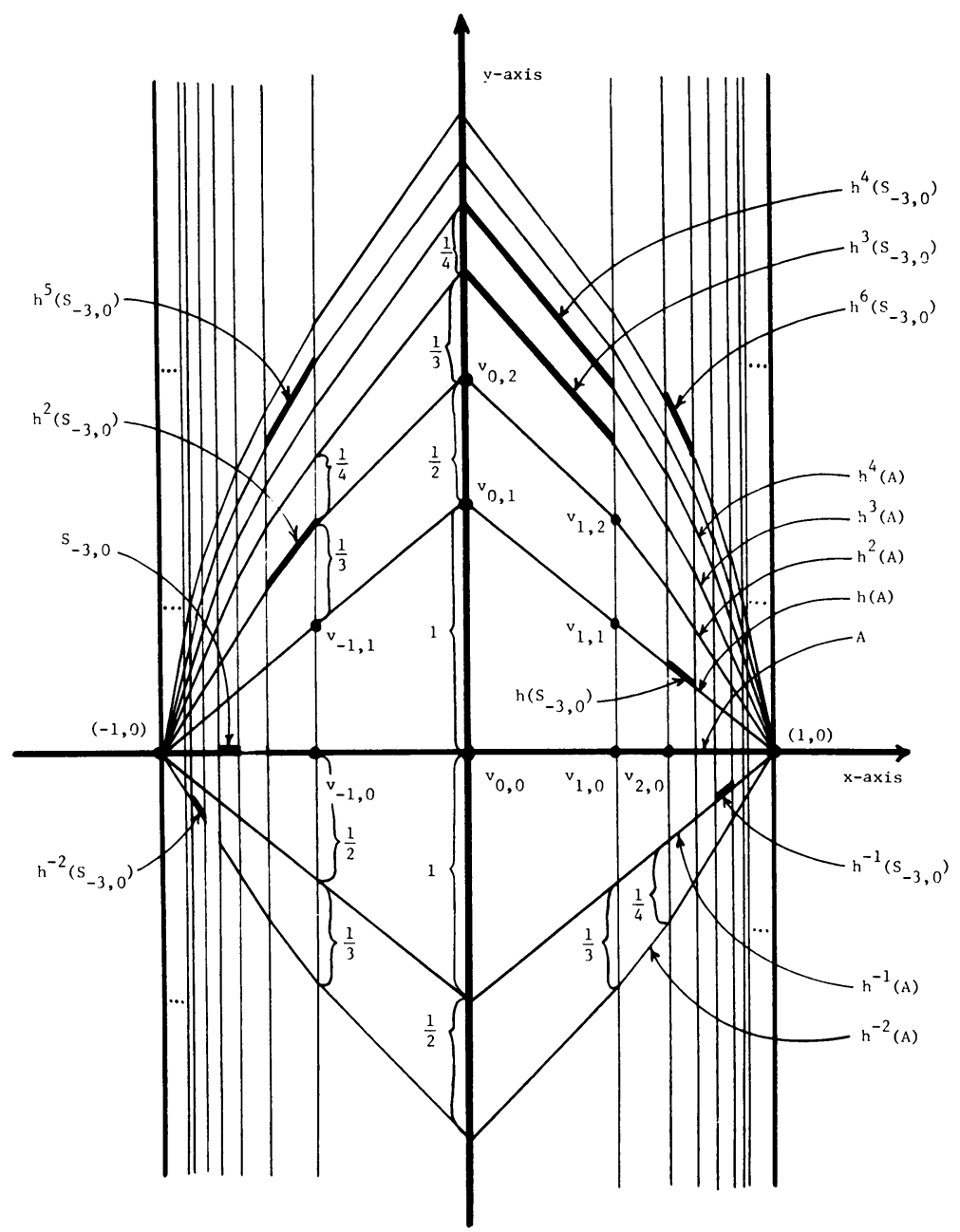

FIGURE 2

For all $j$ and $k \geqslant 1$, extend $h$ linearly to a homeomorphism on the segments $S_{j, k}^{(n-1) / n}, n \geqslant 2$, and $S_{j, k}^{1 / n}, n \geqslant 3$, by defining

$$
\begin{aligned}
h\left(S_{j, k}^{(n-1) / n}\right) & =h\left(\left[v_{j-1, k}^{(n-1) / n}, v_{j, k}^{(n-1) / n}\right]\right) \\
& \equiv\left[h\left(v_{j-1, k}^{(n-1) / n}\right), h\left(v_{j, k}^{(n-1) / n}\right)\right],
\end{aligned}
$$

and

$$
h\left(S_{j, k}^{1 / n}\right)=h\left(\left[v_{j-1, k}^{1 / n}, v_{j, k}^{1 / n}\right]\right) \equiv\left[h\left(v_{j-1, k}^{1 / n}\right), h\left(v_{j, k}^{1 / n}\right)\right] .
$$

We define $h$ on the region between $A_{k,(n-1) / n}$ and $A_{k, n /(n+1)}$, where $n \geqslant 2$, by mapping each vertical segment $[p, q]$ joining $A_{k,(n-1) / n}$ to $A_{k, n /(n+1)}$ linearly onto the vertical segment $[h(p), h(q)]$. Define $h$ on the region between $A_{k, 1 /(n+1)}$ and $A_{k, 1 / n}$, where $n \geqslant 4$, as above, using all vertical segments joining $A_{k, 1 /(n-1)}$ to $A_{k, 1 / n}$. Refer to Figure 3 for $k$ odd. 


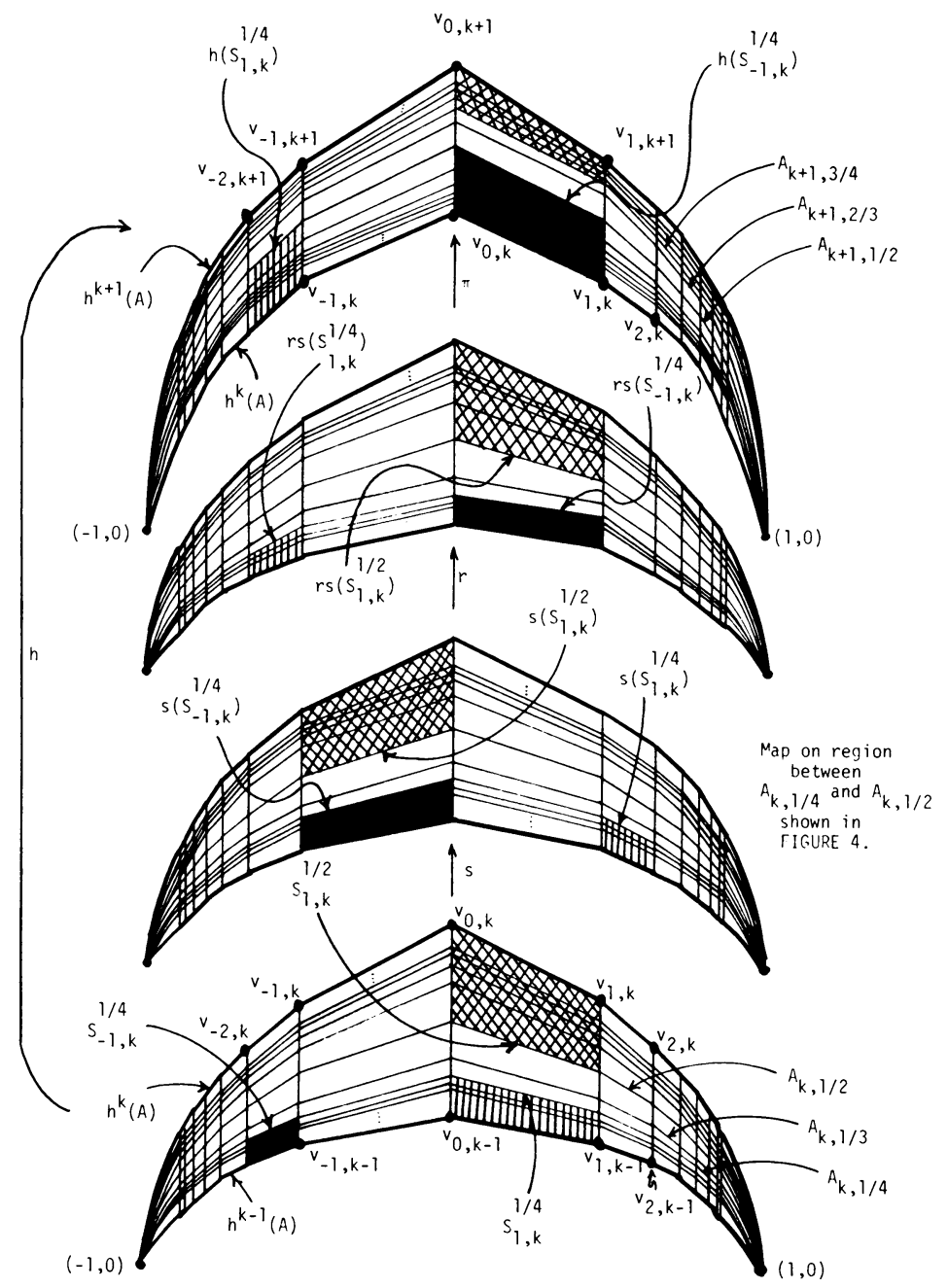

FIGURE 3

We map the region between $A_{k, 1 / 4}$ and $A_{k, 1 / 2}$ to the region between $A_{k+1,1 / 2}$ and $A_{k+1,3 / 4}$ as follows: for all $j, k \geqslant 1$, and $n=3,4$, we define $h$ as a linear map so that

$$
\begin{aligned}
& h\left(\left[v_{j-1, k}^{1 /(n-1)}, v_{j, k}^{1 /(n-1)}, v_{j+\left(-1+(-1)^{k}\right) / 2, k}^{1 / n}\right]\right) \\
& \equiv\left[h\left(v_{j-1, k}^{1 /(n-1)}\right), h\left(v_{j, k}^{1 /(n-1)}\right), h\left(v_{j+\left(-1+(-1)^{k}\right) / 2, k}^{1 / n}\right)\right],
\end{aligned}
$$

and

$$
\begin{aligned}
& h\left(\left[v_{j-1, k}^{1 / n}, v_{j, k}^{1 / n}, v_{j-\left(1+(-1)^{k}\right) / 2, k}^{1 / n-1)}\right]\right) \\
& \equiv\left[h\left(v_{j-1, k}^{1 / n}\right), h\left(v_{j, k}^{1 / n}\right), h\left(v_{j-\left(1+(-1)^{k}\right) / 2, k}^{1 /(n-1)}\right)\right] .
\end{aligned}
$$

For odd $k$ refer to Figure 4. 


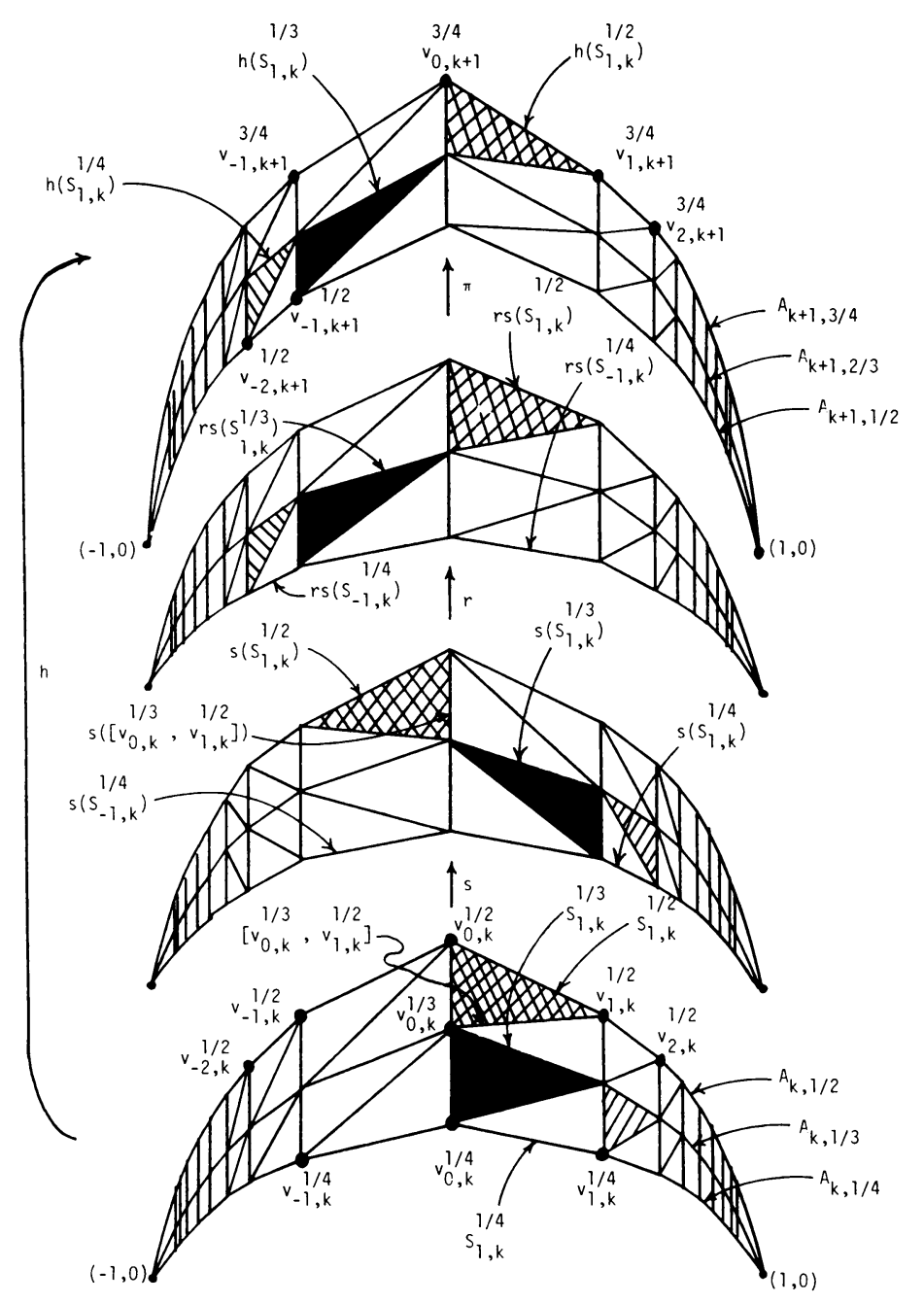

Figure 4

Observe that, for $n \geqslant 2, h\left(A_{k, 1 /(n+2)}\right)=A_{k+1,1 / n}$ and $h\left(A_{k,(n-1) / n}\right)=$ $A_{k+1,(n+1) /(n+2)}$. Note also that the image under $h$ of a vertical segment joining $A_{k, 1 / 2}$ to $h^{k}(A)$ is a vertical segment joining $A_{k+1,3 / 4}$ to $h^{k+1}(A)$. Similarly, a vertical segment joining $h^{k-1}(A)$ to $A_{k, 1 / 4}$ is mapped under $h$ to a vertical segment joining $h^{k}(A)$ to $A_{k+1,1 / 2}$. On the region between $A_{k, 1 / 4}$ and $A_{k, 1 / 2}, h$ may also be viewed as the composition of three maps: a shift $s$, a reflection $r$ through the $y$-axis, and an upward projection $\pi$ to the region between $A_{k+1,1 / 2}$ and $A_{k+1,3 / 4}$. Note that when $k$ is odd $A_{k, 1 / 4}$ shifts "right" and $A_{k, 1 / 2}$ shifts "left"; the reverse occurs when $k$ is even; $s$ is the identity on $A_{k, 1 / 3}$ whether $k$ is odd or even (Figure 4).

For a point $p$ in $B$ on or below the reflection of $h(A)$ through the $x$-axis, define $h(p)=-h^{-1}(-p)$. We extend $h$ linearly on the region between $h^{-1}(A)$ and $A$ by sending every vertical segment $[p, q]$ joining $h^{-1}(A)$ to $A$ to the segment $[h(p), h(q)]$. 
4. $h$ is fixed point free. Clearly no point in the complement of $B$ remains fixed under $h$. For every $k, h$ is fixed point free on $h^{k}(A)$ since the intersection of $h^{k}(A)$ and $h^{k+1}(A)$ is empty. If $p$ is a point between $h^{k-1}(A)$ and $h^{k}(A)$, then $h(p)$ is between $h^{k}(A)$ and $h^{k+1}(A)$; thus, $p \neq h(p)$.

5. Every point has bounded orbit. If $p$ is in the complement of $B$, then $O(p)=$ $\{p, h(p)\}$.

Observe that

$$
\begin{aligned}
h^{n}\left(v_{k, 0}\right) & =h^{n}(k /(k+1), 0) \\
& \equiv\left(\frac{(-1)^{n}(n+k)}{n+k+1}, \sum_{i=n+k+1}^{2 n+k} \frac{1}{i}\right), \text { for } n, k \geqslant 1,
\end{aligned}
$$

and

$$
\begin{aligned}
h^{n}\left(v_{-k, 0}\right) & =h^{n}(-k /(k+1), 0) \\
& \equiv\left(\frac{(-1)^{n}(n-k)}{n-k+1}, \sum_{i=n-k+1}^{2 n-k} \frac{1}{i}\right), \text { for } k \geqslant 1, n \geqslant k .
\end{aligned}
$$

Since $\left(\sum_{i=1}^{n} 1 / i-\log n\right) \rightarrow \gamma$, where $\gamma$ is the Euler constant, one can show that, for fixed $k$,

$$
\lim _{n \rightarrow \infty} \sum_{i=n+k+1}^{2 n+k} \frac{1}{i}=\lim _{n \rightarrow \infty} \sum_{i=n-k+1}^{2 n-k} \frac{1}{i}=\log 2 .
$$

Hence, for every $j$, the positive orbit of $v_{j, 0}$ is bounded. If $p$ is in $\left[v_{j-1,0}, v_{j, 0}\right]$, then $h^{n}(p)$ is in $\left[h^{n}\left(v_{j-1,0}\right), h^{n}\left(v_{j, 0}\right)\right]$ implies that the positive orbit of every point on $A$ is bounded. Observe that if $p$ is on $h^{k}(A)$, then $h^{-k}(p)$ is on $A$; thus, the positive orbit of every point on $h^{k}(A)$ is bounded for every $k \geqslant 0$.

To check points between $h^{k-1}(A)$ and $h^{k}(A)$, for $k>1$, we first consider a point $p$ in the region above $A_{k, 1 / 2}$ and below $h^{k}(A)$. Let $q$ be the point on $h^{k}(A)$ which is directly above $p$ (i.e. $q$ is the intersection of $h^{k}(A)$ with the vertical line containing $p$ ). Since $q$ has bounded positive orbit and $h^{n}(p)$ is directly below $h^{n}(q)$, for all $n \geqslant 0$, the positive orbit of $p$ must also be bounded. Next suppose $p$ is between $A_{k, 1 /(n+1)}$ and $A_{k, 1 / n}$, where $n \geqslant 2$. By construction there is a positive integer $m$ such that $h^{m}(p)$ is between $A_{k+m, 1 / 2}$ and $h^{k+m}(A)$. Thus, the above argument shows that $p$ has a bounded positive orbit. In fact, since a point between $h^{-k}(A)$ and $h^{-k+1}(A), k \geqslant 0$, is between $A$ and $h(A)$ after $k$ applications of $h$, all points in $B$ have bounded positive orbits.

One can see that the negative orbits of points in $B$ are bounded by recalling that $h^{-1}(p)=-h(-p)$.

6. $h$ is a homeomorphism. It follows from the construction that $h$ is bijective, and that $h$ is continuous at every point $(x, y)$ with $|x| \neq 1$. To show that $h$ is continuous on the lines $x= \pm 1$, we first consider a point $(-1, y)$, where $y \geqslant 0$. Since $h$ is a reflection on the left side of the line $x=-1$, it suffices to show that if $\left(x_{m}, y_{m}\right) \rightarrow$ $(-1, y)$, then $h\left(x_{m}, y_{m}\right) \rightarrow(1, y)$, where $\left\{\left(x_{m}, y_{m}\right)\right\}$ is contained in $B$. Observe that $B$ is partitioned into convex polygons bounded by the lines $x= \pm n /(n+1)$ and the $\operatorname{arcs} h^{k}(A)$, where $n$ and $k$ are integers. Thus, for $m$ sufficiently large, $\left(x_{m}, y_{m}\right)$ is 
contained in a quadrilateral bounded by the lines

$$
x=-n_{m} /\left(n_{m}+1\right), \quad x=-\left(n_{m}-1\right) / n_{m},
$$

and the $\operatorname{arcs} h^{k_{m}-1}(A)$ and $h^{k_{m}}(A)$, where $n_{m} \geqslant 4$. Hence,

$$
\frac{-n_{m}}{n_{m}+1} \leqslant x_{m} \leqslant-\frac{n_{m}-1}{n_{m}} \text { and } \sum_{i=n_{m}+1}^{n_{m}+k_{m}+1} \frac{1}{i} \leqslant y_{m} \leqslant \sum_{i=n_{m}}^{n_{m}+k_{m}+1} \frac{1}{i} \text {. }
$$

Let $h\left(x_{m}, y_{m}\right)=\left(x_{m}^{\prime}, y_{m}^{\prime}\right)$. By viewing the image of the quadrilateral one can see that

$$
\frac{n_{m}-2}{n_{m}-1} \leqslant x_{m}^{\prime} \leqslant \frac{n_{m}+1}{n_{m}+2} \text { and } \sum_{i=n_{m}+2}^{n_{m}+k_{m}+3} \frac{1}{i} \leqslant y_{m}^{\prime} \leqslant \sum_{i=n_{m}-1}^{n_{m}+k_{m}+1} \frac{1}{i} \text {. }
$$

Since $x_{m} \rightarrow-1, n_{m}$ must increase without bound; thus, $x_{m}^{\prime} \rightarrow 1$. To see that $y_{m}^{\prime} \rightarrow y$ observe that

$$
\sum_{i=n_{m}+1}^{n_{m}+k_{m}+1} \frac{1}{i}-\sum_{i=n_{m}-1}^{n_{m}+k_{m}+1} \frac{1}{i} \leqslant y_{m}-y_{m}^{\prime} \leqslant \sum_{i=n_{m}}^{n_{m}+k_{m}+1} \frac{1}{i}-\sum_{i=n_{m}+2}^{n_{m}+k_{m}+3} \frac{1}{i} .
$$

The differences of the sums on each side of the bound decrease to 0 as $m \rightarrow \infty$; hence, $y_{m}-y_{m}^{\prime} \rightarrow 0$ implies that $y_{m}^{\prime} \rightarrow y$. The arguments for the remaining points of form $( \pm 1, \pm y)$ are analogous to the argument given above.

7. COROLlary. There exist fixed point free orientation preserving and orientation reversing homeomorphisms of $E^{3}$ onto itself such that the orbit of every point is bounded.

Proof. The homeomorphism $f$ defined by $f(x, y, z)=\left(x^{\prime}, y^{\prime}, z\right)$, where $\left(x^{\prime}, y^{\prime}\right)=$ $h(x, y)$ and $h$ is the homeomorphism constructed above, verifies the corollary for the orientation reversing case. For the orientation preserving case simply define $g(x, y, z)=\left(x^{\prime}, y^{\prime},-z\right)$, where $\left(x^{\prime}, y^{\prime}\right)=h(x, y)$.

\section{BIBLIOGRAPHY}

1. Stephen Andrea, On homeomorphisms of the plane which have no fixed points, Abh. Math. Sem. Univ. Hamburg 30 (1967), 61-74. MR 34 \#8397.

2. The plane is not compactly generated by a free mapping, Trans. Amer. Math. Soc. 151 (1970), 481-498. MR 42 \#2445.

3. Harold Bell, On fixed point properties of plane continua, Trans. Amer. Math. Soc. 128 (1967), 539-548. MR 35 \# 4888.

4. R. H. Bing, The elusive fixed point property, Amer. Math. Monthly 76 (1969), 119-132. MR 38 \# 5201 .

5. B. L. Brechner and R. D. Mauldin, Homeomorphisms of the plane, Pacific J. Math. 59 (1975), 375-381. MR 52 \#9199.

6. $\ldots \mathrm{EC}^{+}$homeomorphisms of Euclidean spaces, Topology Proc. 1 (1976), 335-343.

7. L. E. J. Brouwer, Beweis des ebenen Translationesatzes, Math. Ann. 72 (1912), 39-54.

8. Gordon Johnson, An example in fixed point theory, Proc. Amer. Math. Soc. 44 (1974), 511-514.

9. B. v. Kerekjarto, On a geometric theory of continuous groups, Ann. Math. 26 (1925), $105-117$.

10. E. v. Sperner, Uber die fixpunktfreien Abbildungen der Ebene, Abh. Math. Sem. Univ. Hamburg 10 (1934), 1-47.

11. H. Terasaka, On quasi translations in $E^{n}$, Proc. Japan Acad. Ser. A Math. Sci. 30 (1954), 80-84.

12. S. M. Ulam, Problems in modern mathematics, Wiley, New York, 1960. MR 22 \# 10884.

Department of Mathematics, University of Florida, Gainesville, Florida 32611

Current address: 2B630A, Bell Laboratories, Holmdel, New Jersey 07733 